J. Lake Sci.(湖泊科学) , 2018, 30(3):693-700

DOI 10. 18307/2018. 0311

(c) 2018 by Journal of Lake Sciences

\title{
大型浅水湖泊藻类模型参数敏感性分析”
}

\author{
姜 龙 ${ }^{1,2}$, 李一平 ${ }^{1,2 * *}$, 章双双 ${ }^{3}$, 王文才 ${ }^{4}$, 翁晟琳 ${ }^{1,2}$, 杜 微 $^{5}$, 王建威 ${ }^{6}$ \\ (1: 河海大学浅水湖泊综合治理与资源开发教育部重点实验室, 南京 210098) \\ (2:河海大学环境学院, 南京 210098) \\ (3:苏州科技大学,苏州 215009) \\ (4:环境保护部华南环境科学研究所,广州 510655) \\ (5:环境保护部南京环境科学研究所,南京 210042) \\ (6: 中国电建集团华东勘测设计研究院有限公司,杭州 310014)
}

\begin{abstract}
摘 要: 选取太湖作为典型湖泊在之前研究基础上建立藻类模型, 对模型中与藻类有关的 40 个参数进行拉丁超立方抽 样, 并使用区域敏感性分析方法和普适似然不确定性分析方法进行敏感性分析. 结果表明: 在所选的 40 个参数中, 有 7 个参数是敏感的参数, 对模拟的结果影响较大. 在藻类生长、基础代谢、牧食和沉降 4 个藻类变化过程中藻类生长的敏感 参数最多, 影响最大; 在藻类生长项中, 叶绿素的消光系数是藻类生长光照限制中的最敏感参数, 而最低适宜生长温度及 其对藻类生长的影响系数则是温度限制中的敏感参数; 并且不同湖区的不确定性在不同时间差异明显, 对于藻类低浓度 湖区和藻类暴发期的模拟需要加以关注.
\end{abstract}

关键词: 太湖; 区域敏感性分析方法 (RSA); 普适似然不确定性分析方法 (GLUE) ; 参数敏感性;藻类模型

\section{Parameter sensitivity analysis of algal model in large shallow lakes}

JIANG Long $^{1,2}$, LI Yiping ${ }^{1,2 * *}$, ZHANG Shuangshuang ${ }^{3}$, WANG Wencai ${ }^{4}$, WENG Shenglin ${ }^{1,2}$, DU Wei ${ }^{5} \&$ WANG Jianwei ${ }^{6}$

(1: Key Laboratory of Integrated Regulation and Resource Development on Shallow Lakes, Ministry of Education, Hohai University, Nanjing 210098, P.R.China)

(2: College of Environment, Hohai University, Nanjing 210098, P.R.China)

(3: Suzhou University of Science and Technology, Suzhou 215009, P.R.China)

(4: South China Institute of Environmental Science, Guangzhou 510655, P.R.China)

(5: Nanjing Institute of Environmental Science, Ministry of Environmental Protection, Nanjing 210042, P.R.China)

(6: HydroChina Huadong Engineering Corporation, Hangzhou 310014, P.R. China)

\begin{abstract}
Lake Taihu was chosen as it is a typical large shallow lake, and algal model was established. 40 parameters in water quality model of the lake were filtered by previous research and sampled with Latin hypercube sampling method, and regionalized sensitivity analysis method and generalized likelihood uncertainty estimation method applied for uncertainty and sensitivity analysis, respectively. The results show that only 7 parameters selected were sensitive, and algal growth was the most important factor between the growth, basic metabolism, predation and settlement of algae. The extinction coefficient of chlorophyll was the most sensitive parameters in the light limitation of algae growth, and suboptimal temperature effect coefficient for growth also turned to be sensitive in the temperature limitation. In addition, uncertainty distinguished in different areas and period, more attention should be paid on low concentration areas and periods of algal blooms.
\end{abstract}

Keywords: Lake Taihu; regionalized sensitivity analysis; generalized likelihood uncertainty estimation; parameter sensitivity;

* 国家自然科学基金项目 $(5177090079,51579071,51539003)$ 、国家重点研发计划项目(2016YFC0401703)和创新研 究群体科学基金项目 (51421006) 联合资助. 2017-09-13 收稿; 2017-10-17 收修改稿. 姜龙 (1993 ), 男,硕士研 究生;E-mail: ziyamubo@hhu.edu.cn.

** 通信作者;E-mail: liyiping@ hhu.edu.cn. 
algal model

湖泊作为一种常见且重要的水体, 近年来受到气候变化和人类活动的影响, 许多湖泊富营养化问题非 常严重, 水生态环境也持续恶化. 藻类模型作为湖泊环境管理决策的一种有效工具被广泛应用和研究 ${ }^{[1]}$, 但 是由于模型受到各种外界因素的干扰, 存在着不可避免的不确定性. 为了提高模型的模拟精度, 增强模型模 拟的科学合理性,需要对水质模型的不确定性来源进行分析.

模型的不确定性来源大致可以分为结构、输人数据、参数以及计算误差 4 类 ${ }^{[2]}$. 近年来随着计算机技术 的发展, 各种水环境模型结构更加完善, 但是同时参数也更多, 参数的影响变得非常重要, 尤其是敏感性参 数对于模拟结果不确定性贡献非常大. 大量关于湖泊水环境模型敏感性分析的研究表明与生物生长代谢以 及死亡速率有关的参数都较敏感 ${ }^{[3-7]}$. 同时敏感性参数会随着湖泊大小和深度呈现差异, 尤其是一些易受风 浪和边界条件影响的大型浅水湖泊, 在这些水体中水动力以及浮游藻类生长过程非常复杂, 光照、温度以及 沉降速度等因素对模型的模拟结果影响也较大. 例如在威尼斯泻湖模型中, 光照强度以及磷半饱和常数较 为敏感 ${ }^{[8-9]}$. 而在滇池模型中, 硝化速率以及光削减系数较为敏感 ${ }^{[10]}$. 但是对于太湖这样面大水浅、水环境 复杂的淡水湖泊, 藻类模型敏感性研究较少, 对于大型富营养化浅水湖泊模型的参数敏感性认识不足.

本研究选取太湖作为典型的大型浅水湖泊进行分析, 选取国内外常用的 EFDC ( environmental fluid dynamic code) 模型, 结合已有数据资料建立太湖藻类模型并选取叶绿素作为模型输出. 本研究利用区域敏感 性分析方法 (regionalized sensitivity analysis, RSA) 和普适似然不确定性分析方法 (generalized likelihood uncertainty estimation, GLUE) 对参数随机取样并进行敏感性分析, 量化参数对模拟结果的影响和每个参数的 敏感性, 并通过与后验分布得到参数可能的取值范围. 研究结果能够帮助对比分析这类湖泊的参数敏感性 特点, 提高参数调整的效率和模型模拟精度, 为选取较优、较符合实际的湖泊藻类模型参数提供参考.

\section{1 研究区域}

本文以中国第三大浅水湖泊太湖 $\left(30^{\circ} 05^{\prime} \sim 32^{\circ} 08^{\prime} \mathrm{N}, 119^{\circ} 08^{\prime} \sim 122^{\circ} 55^{\prime} \mathrm{E}\right)$ 为研究对象. 太湖总面积 2338 $\mathrm{km}^{2}$, 平均水深 $1.9 \mathrm{~m}$, 最大水深为 $2.6 \mathrm{~m}$. 太湖流域夏季主导风向是东南风, 冬季主导风向是西北风, 平均风 速 $3.5 \sim 5.0 \mathrm{~m} / \mathrm{s}$. 太湖底部为坡度为 $0^{\circ} 0^{\prime} 19.66^{\prime \prime}$ 的平底地形, 周边水系复杂, 本研究将环湖河道概化为 30 条 河 ${ }^{[11]}$, 编号如图 1 所示. 由于水动力条件较弱, 营养盐浓度较高, 因此太湖在夏、秋季节常暴发藻类水华, 并 且存在着较大的空间差异性, 其中竺山湾和梅梁湾最为严重, 藻类最多, 东部沿岸区最少 ${ }^{[12]}$. 为方便分析, 本研究将太湖划分为 8 个湖区 (图 1), 其中 4 个典型湖区 (梅梁湾、西南湖区、湖心区和东部湖区) 在地理位 置上包含了湖心 (湖心区)、湖湾 (梅梁湾和东部湖区) 和湖岸 (西南湖区) ; 在水动力情况上包含了以湖流 (东部湖区) 和波浪 (其他湖区) 为主的湖区; 在水质情况上包含了高营养盐负荷和高藻类浓度的梅梁湾, 中 营养盐负荷和中藻类浓度的湖心区以及低营养盐负荷和低藻类浓度的东部湖区, 因此选取这 4 个典型湖区 进行参数的敏感性分析.

\section{2 研究方法}

\section{1 水质模型概况}

选取国内外常用的 EFDC 模型来模拟太湖的水动力和水质过程. 采用笛卡尔直角坐标划分网格, 共划 分 4464 个网格, 每个网格单元边长为 $750 \mathrm{~m}$, 垂直方向采用 $\sigma$ 坐标将太湖分为 3 层 ${ }^{[13]}$. 为满足流体静力学 的连续性和避免产生 $\sigma$ 坐标带来的压力梯度错误, 通过改变网格密度和插值方式使模型湖底坡度小于 $0.33^{[11]}$. 模型使用大气条件、表面风力、出人湖的流量和水质作为边界条件. 其中边界河道输人条件来自太 湖周边的大小出人河流概化成的 30 条主要河流 (图 1); 降雨量数据来自太湖附近 8 个监测站, 大气条件和 风场采用中国科学院南京地理与湖泊研究所太湖湖泊生态系统国家野外观测研究站的数据. 模拟年份为 2005 年, 太湖平均风速为 $5 \mathrm{~m} / \mathrm{s}$, 年平均气温为 $17.7^{\circ} \mathrm{C}$, 年平均降雨量为 $1833 \mathrm{~mm}$, 平均太阳辐射为 $245 \mathrm{~W} / \mathrm{m}^{2}$, 平均云覆盖系数为 0.6 . 模拟初始日期为 1 月 1 日, 假设初始水位为水平, 设置为模拟时段第 $1 \mathrm{~d}$ 的平均值, 初始流速设为 $0 \mathrm{~m} / \mathrm{s}^{[13]}$. 模型采用稳定的初始条件和边界条件, 计算时间为 $365 \mathrm{~d}$, 时间步长为 $10 \mathrm{~s}$. 模型参 


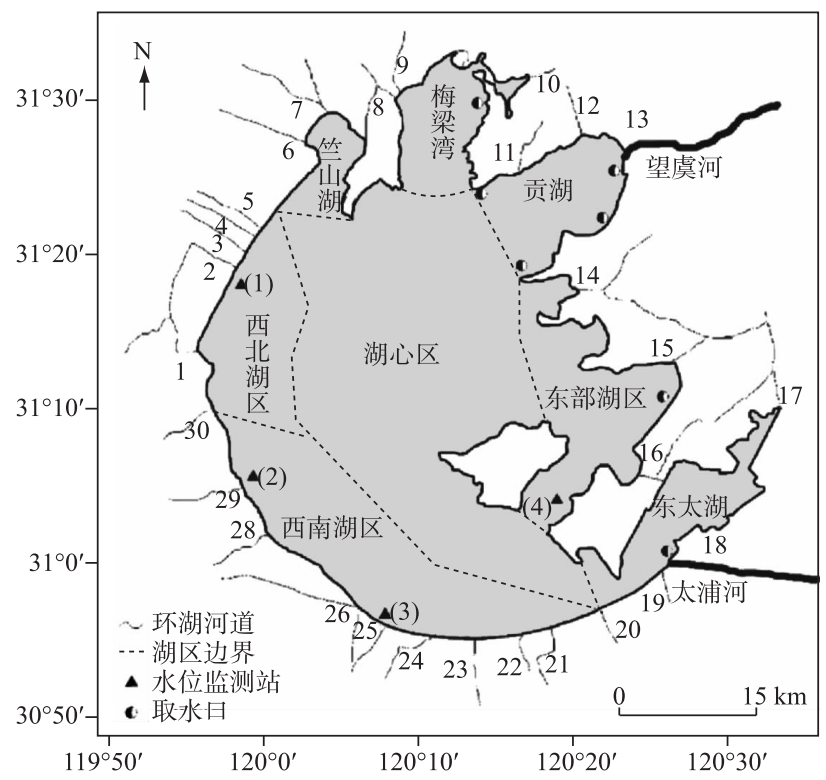

图 1 研究区域划分

Fig. 1 Division of the study areas

数的设置已经使用实测值进行了率定 ${ }^{[11,13-14]}$.

\section{2 分析方法}

采用区域敏感性分析方法 RSA 和普适似然不确定性分析方法 GLUE 来分析参数的敏感性. RSA 方法是 由 Hornberger 等提出的 ${ }^{[15]}$, 通过比较每组参数所得到的模拟值与实测值, 获得参数组的行为 (即拟合度较 高) 和非行为 (即拟合度较低) 特征, 并计算参数的累计分布. RSA 方法具有对最优条件进行弱化并使用一 些定量或定性方式来决定参数取舍的特点 ${ }^{[16]}$. GLUE 方法是 Beven 等基于 RSA 方法提出的用于评价预测结 果不确定性的全局方法, 是一个 Monte Carlo 方法 ${ }^{[17]}$. 这两种方法的基本原理是首先将参数抽样后代人模型 运行, 计算每个模拟结果与实测值的似然值, 设定阈值为 0.5 , 将似然值低于该阈值的参数组篮除, 把似然值 高于该阈值的重新进行归一化, 最后按照似然值的大小排序, 求出在某置信度下模型预报的不确定性范围 并分析参数的敏感性. 由于在实际监测中测量指标大多为叶绿素, 为了方便比较, 本研究中采用可以表征藻 类含量的叶绿素作为目标函数进行分析. 具体步骤如下:

1) 确定参数的取值范围和先验分布

根据文献中水质模型的参数设置 ${ }^{[18-21]}$, 并针对大型浅水湖泊的特点, 在选取参数时首先考虑到蓝藻是 太湖的优势藻类, 因此去除了模型中反映其他藻类 (如绿藻、硅藻和其他大型浮游藻类) 生长特性的参数, 如 藻类生长率、藻类基础代谢速率、藻类牧食速率等. 在预模拟实验中也发现太湖的内源释放速率对于营养盐 模拟影响十分大, 但是由于本模型主要研究的是水质模块中与藻类有关的参数, 模型的沉积模块并没有开 启, 水质模块中的内源释放更类似于外部输人条件而不是参数, 因此不考虑. 另外, 参考温度、藻类生长最佳 水深和其他通用的参数也被笁除, 再根据剩余参数的计算方程, 选取了方程的一阶反应项系数、沉降项等. 最终选择蓝藻最大生长速率、蓝藻基础代谢速率等 40 个参数作为水质模型输人参数. 假定各个参数符合均 匀分布,且参数之间取值相互独立, 模型参数、意义及其取值范围见表 1 .

2) 模拟计算

对于拉丁超立方抽样 (LHS) 的次数, 本研究对 $100 、 200 、 300 、 400$ 和 500 次 5 种情况进行了鲁棒性检验, 发现当抽样次数大于 400 次时, 抽样运行结果保持稳定. 故使用 LHS 方法进行随机抽样, 生成 500 组参数, 运行模型, 得到 500 组输出结果. 
表 1 模型输人参数抽样取值范围

Tab.1 Parameters used for the Latin hypercube sampling

\begin{tabular}{|c|c|c|c|c|c|}
\hline 参数组 & 参数 & 参数意义 & 单位 & 最小值 & 最大值 \\
\hline \multirow[t]{3}{*}{ 藻类生长 } & PMc & 蓝藻最大生长速率 & $d^{-1}$ & 2 & 5 \\
\hline & BMRc & 蓝藻基础代谢速率 & $d^{-1}$ & 0.01 & 0.06 \\
\hline & PRRc & 蓝藻牧食速率 & $d^{-1}$ & 0.01 & 0.06 \\
\hline 硝化 & rNitM & 最大硝化率 & $d^{-1}$ & 0.04 & 0.2 \\
\hline 溶解氧 & KRO & 复氧速率常数 & - & 1.5 & 5.32 \\
\hline 化学需氧量 & KCD & 化学需氧量衰减速率 & $d^{-1}$ & 0.01 & 0.15 \\
\hline \multirow[t]{9}{*}{ 水解和矿化 } & KRN & 难溶颗粒态有机氮最小水解速率 & $d^{-1}$ & 0.001 & 0.01 \\
\hline & KLN & 活性颗粒态有机氮最小水解速率 & $d^{-1}$ & 0.01 & 0.1 \\
\hline & KDN & 溶解态有机氮最小矿化速率 & $d^{-1}$ & 0.01 & 0.08 \\
\hline & KRC & 难溶颗粒态有机碳最小水解速率 & $d^{-1}$ & 0.001 & 0.01 \\
\hline & KLC & 活性颗粒态有机碳最小水解速率 & $d^{-1}$ & 0.01 & 0.1 \\
\hline & KDC & 溶解态有机碳最小矿化速率 & $d^{-1}$ & 0.005 & 0.15 \\
\hline & KRP & 难溶颗粒态有机磷最小水解速率 & $d^{-1}$ & 0.001 & 0.01 \\
\hline & KLP & 活性颗粒态有机磷最小水解速率 & $d^{-1}$ & 0.01 & 0.1 \\
\hline & KDP & 溶解态有机磷最小矿化速率 & $d^{-1}$ & 0.01 & 0.3 \\
\hline \multirow[t]{4}{*}{ 光照 } & Keb & 背景消光系数 & $\mathrm{m}^{-1}$ & 0.45 & 0.55 \\
\hline & KeTSS & 悬浮颗粒物消光系数 & $\mathrm{L} /(\mathrm{m} \cdot \mathrm{mg})$ & 0.01 & 0.1 \\
\hline & KeChl & 叶绿素消光系数 & $\mathrm{L} /(\mathrm{m} \cdot \mathrm{mg})$ & 0.01 & 0.07 \\
\hline & IsMIN & 最小合适太阳辐射 & Langley/d & 40 & 60 \\
\hline \multirow[t]{7}{*}{ 半饱和常数 } & KHNitDO & 硝化氧半饱和常数 & $\mathrm{g} \mathrm{O}_{2} / \mathrm{m}^{3}$ & 0.5 & 1 \\
\hline & KHNitN & 硝化氨半饱和常数 & $\mathrm{g} \mathrm{N} / \mathrm{m}^{3}$ & 0.5 & 1 \\
\hline & KHCOD & 化学需氧量衰减氧半饱和常数 & $\mathrm{mg} / \mathrm{L} \mathrm{O}_{2}$ & 1 & 1.5 \\
\hline & KHNc & 蓝藻生长氮半饱和常数 & $\mathrm{mg} / \mathrm{L}$ & 0.01 & 0.25 \\
\hline & KHPc & 蓝藻生长磷半饱和常数 & $\mathrm{mg} / \mathrm{L}$ & 0.001 & 0.005 \\
\hline & KHDNN & 反硝化半饱和常数 & g N/m $\mathrm{m}^{3}$ & 0.05 & 0.2 \\
\hline & KHORDO & 藻类呼吸氧半饱和常数 & $\mathrm{g} \mathrm{O}_{2} / \mathrm{m}^{3}$ & 0.5 & 2 \\
\hline \multirow[t]{11}{*}{ 温度 } & KTHDR & 水解温度影响系数 & - & 0.05 & 0.1 \\
\hline & KTMNL & 矿化温度影响系数 & - & 0.05 & 0.1 \\
\hline & KTCOD & 化学需氧量衰减温度速率常数 & - & 0.03 & 0.05 \\
\hline & KNit1 & 温度低于最适硝化温度对硝化率的影响系数 & - & 0.002 & 0.006 \\
\hline & KNit2 & 温度高于最适硝化温度对硝化率的影响系数 & - & 0.002 & 0.006 \\
\hline & KTG1c & 温度低于 TMc1 时对蓝藻群体生长的影响 & - & 0.001 & 0.01 \\
\hline & KTG2c & 温度高于 TMc2 时对蓝藻群体生长的影响 & - & 0.001 & 0.01 \\
\hline & TMc1 & 蓝藻生长适宜温度最小值 & ${ }^{\circ} \mathrm{C}$ & 20 & 27 \\
\hline & TMc2 & 蓝藻生长适宜温度最大值 & ${ }^{\circ} \mathrm{C}$ & 27 & 30 \\
\hline & KTR & 复氧速率的温度调节常数 & - & 1 & 1.05 \\
\hline & $\mathrm{KTBc}$ & 蓝藻基础代谢的温度影响系数 & - & 0.05 & 0.08 \\
\hline \multirow[t]{3}{*}{ 沉降速率 } & WSc & 蓝藻沉降速率 & $\mathrm{m} / \mathrm{d}$ & 0.005 & 0.03 \\
\hline & WSrp & 难溶颗粒态有机物沉降速率 & $\mathrm{m} / \mathrm{d}$ & 0.2 & 1 \\
\hline & WSlp & 活性颗粒态有机物沉降速率 & $\mathrm{m} / \mathrm{d}$ & 0.2 & 1 \\
\hline
\end{tabular}

3) 定义似然判据

选择最常用的 Nash-Stucilffe 确定性系数作为似然判据 ${ }^{[22]}$, 表达式为:

$$
L\left(\theta_{i} / Y\right)=1-\alpha_{i}^{2} / \alpha_{0}^{2}
$$

式中, $L\left(\theta_{i} / Y\right)$ 表示第 $i$ 组参数的似然判据, $\alpha_{i}^{2}$ 表示模拟序列的误差方差, $\alpha_{0}^{2}$ 表示实测序列的方差. 


$$
l\left(\theta_{i} / Y\right)=\frac{L\left(\theta_{i} / Y\right)-L_{\min }\left(\theta_{i} / Y\right)}{L_{\max }\left(\theta_{i} / Y\right)-L_{\min }\left(\theta_{i} / Y\right)}
$$

式中, $l\left(\theta_{i} / Y\right)$ 为归一化后的似然值, $L_{\text {min }}\left(\theta_{i} / Y\right)$ 和 $L_{\text {max }}\left(\theta_{i} / Y\right)$ 分别为最小和最大似然值. 将归一化后的似然 值进行排序, 分别选取 95\% 和 5\%作为 $90 \%$ 置信区间的上下边界分位点. 计算区间覆盖率、不确定区间宽度 和相对不确定宽度等指标评估模型由参数导致的不确定性.

4) 参数敏感性分析

根据在步骤 3) 中得到的似然值大小将参数组分为 10 组, 每组分别计算其累计似然分布, 这些似然值有 总和为 1 的特点. 分析似然值最好的 $10 \%$, 如果在参数分布区间内所得到的累计似然函数是一条直线, 表示 该参数的可识别性较差. 如果累计似然函数偏离较大则表明参数较敏感.

5 ) 参数敏感性量化

对得到的累计似然函数使用 K-S 检验 ${ }^{[23]}$ ( Kolmogorov-Smirnov test). 使用最大垂向偏离度 (Maximum Vertical Distance, $M V D)$ 来定义敏感指数并根据敏感指数的大小将参数分为不同的敏感级别.

\section{3 结果与讨论}

\section{1 参数敏感性}

针对 500 组参数组合使用定义的似然判据计算每一组参数组合对应的似然值, 将似然值按大小分为 10 组后得到在参数取值区间上的累计似然函数图, 通过各个累计似然函数的最大偏离度计算参数敏感性指数. 在 40 个参数中, 敏感参数即 $M V D>0.11, P<0.05$ 的参数有 7 个, 在 4 个湖区的敏感指数如图 2 .

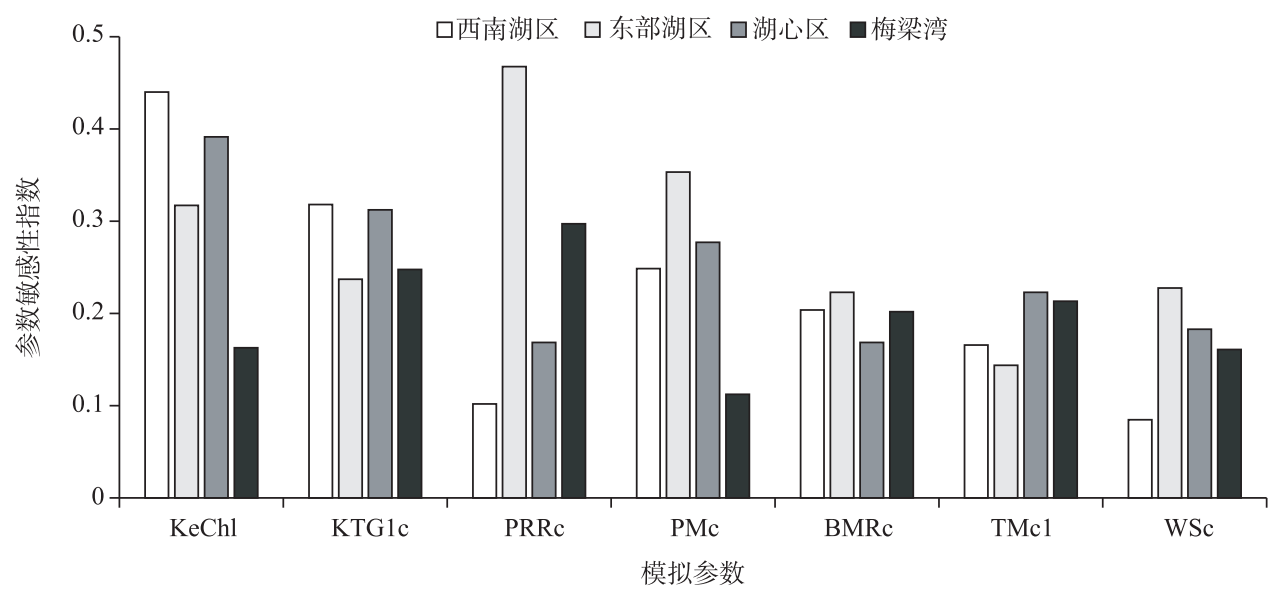

图 2 叶绿素模拟参数敏感性指数 (KeChl: 叶绿素消光系数; KTG1c: 低温时对蓝藻影响系数; $\mathrm{PRRc}$ : 蓝藻牧食速率;PMc: 蓝藻最大生长速率;BMRc:蓝藻基础代谢速率;

$\mathrm{TMc} 1$ : 蓝藻生长适宜温度最小值; WSc : 蓝藻沉降速率)

Fig.2 Sensitive indices in the simulation of chlorophyll

7 个敏感参数可以根据模型的控制方程分为藻类生长项 (KeChl、KTG1c、PMc 和 TMc1)、藻类基础代谢 项 (BMRc) 、藻类牧食项 (PRRc) 和藻类沉降项 (WSc). 其中藻类生长项的敏感参数最多, 这部分的敏感性较 高, 是影响模拟结果的重要因素. 藻类生长参数在其他富营养湖泊如滇池和加里利海中也被发现是至关重 要的 ${ }^{[24-25]}$. 在模型中, 藻类生长项过程较为复杂, 参数也较多, 一般通过限制因子的方式对藻类生长进行描 述. 大致将影响藻类生产速率的限制性因子分为 3 种: 营养盐、光照和温度. KeChl 是光照限制因子中的最敏 感参数, 主要是由于在太湖这样藻类易暴发的浅水湖泊, 水体透明度受藻类的影响非常明显, 而对于一些富 营养化问题较轻的深水湖泊, 背景消光系数则是光照限制因子中的最敏感参数, 如华盛顿湖 ${ }^{[26]}$. KTG1c 和 $\mathrm{TMc} 1$ 被发现是温度限制因子中的最敏感参数, 这主要可能是由于太湖冬、夏季温差较大, 而对于滇池这样 冬、夏季温差较小的大型浅水湖泊模型中 ${ }^{[24]}$, 类似的参数就并不十分敏感. 相比光照和温度等能量因素, 营 
养盐对于模拟的影响较小, 主要是由于太湖本身的营养盐水平较高, 相对于能量因素限制性较小.

表 2 参数敏感性排序

Tab. 2 Sensitivity ranks of parameters

\begin{tabular}{ccccc}
\hline 敏感性排序 & 西南湖区 & 东部湖区 & 湖心区 & 梅梁湾 \\
\hline 1 & KeChl & PRRc & PRRc & KeChl \\
2 & KTG1c & PMc & KTG1c & KTG1c \\
3 & PMc & KeChl & KTBc & PMc \\
4 & BMRc & KTG1c & TMc1 & TMc1 \\
5 & TMc1 & WSc & BMRc & WSc \\
6 & KLP & BMRc & KTG2c & PRRc \\
7 & - & TMc1 & KeChl & BMRc \\
8 & - & - & WSc & - \\
9 & - & - & KNit1 & - \\
10 & - & - & KDC & - \\
11 & - & - & KHPc & - \\
12 & - & - & KHNc & - \\
\hline
\end{tabular}

对于部分参数, 参数敏感性空间变化较大, 如 PRRc 和 KeChl. 将敏感参数在不同湖区进行敏感性 排序 (表 2). 对于营养盐浓度较高的梅梁湾, 与能量 相关的参数 (KeChl 和 KTG1c) 较为敏感, 而东部湖 区营养盐浓度较低, 最大生长速率 $(\mathrm{PMc})$ 和藻类牧 食速率 (PRRc) 最敏感. 对于湖心区, 敏感性参数较 其他的湖区明显得多,这主要可能是湖心区较强的 水动力导致的,水体交换和污染物迁移转化以及藻 类生长过程受到水动力的影响变得活跃, 从而对于 参数变化较为敏感.

综合以上考虑, 在模拟类似太湖这样的面大、水 浅的水动力水质状况复杂的湖泊时, 需要重点调整 这些敏感参数,并且对于营养盐浓度不同的湖区需 要分区考虑, 达到更好的模拟湖泊水动力水质的 目的.

\section{2 模拟结果的不确定性}

4 个湖区的模拟结果对应的置信度为 $90 \%$ 的不确定性区间如图 3 所示, 4 个湖区模拟结果的趋势和实 测值的基本一致，区间覆盖率平均为 79\% , 说明大部分实测值在不确定性区间内, 模型基本可信, 可以用于 太湖水体富营养化模拟.
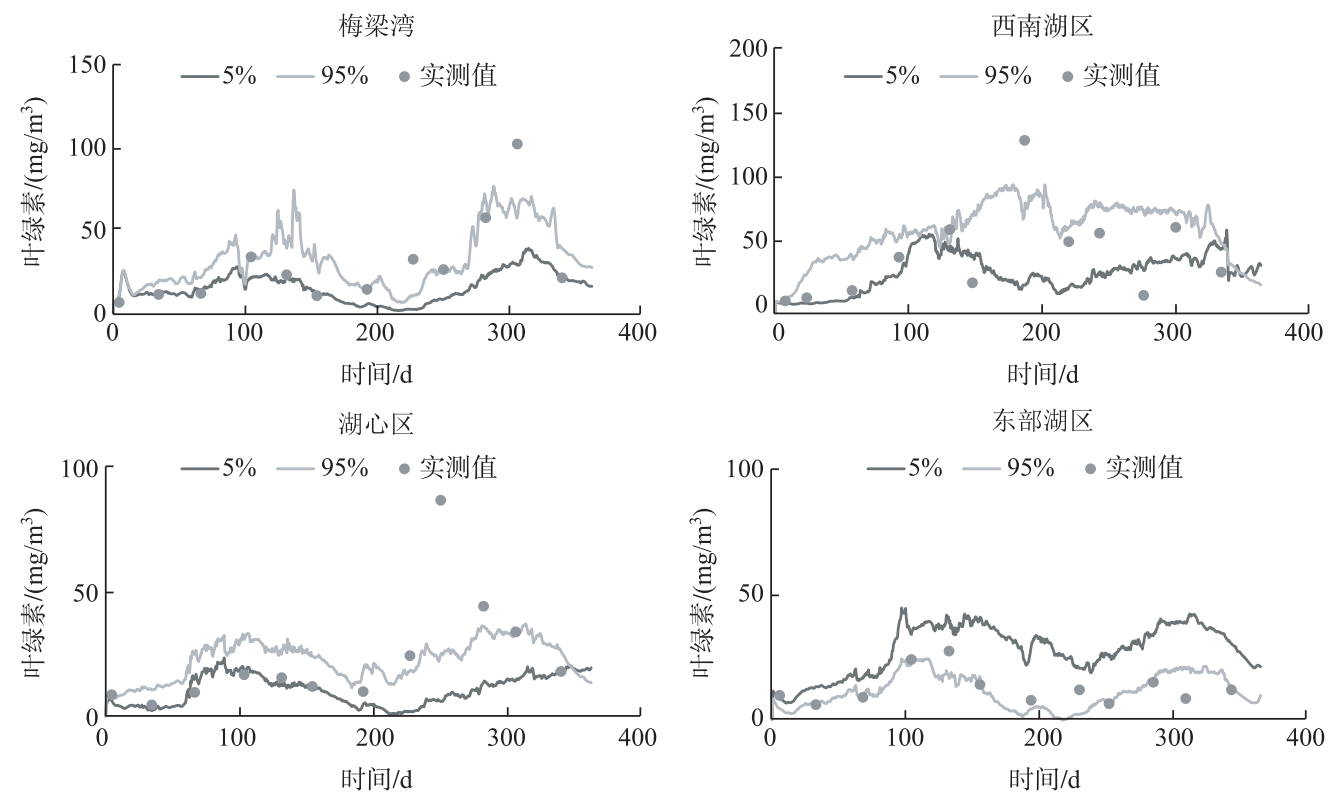

图 34 个湖区叶绿素模拟结果 $90 \%$ 置信区间与实测值

Fig.3 Uncertainty interval and monitoring data of chlorophyll in four subareas

不确定性在不同湖区差异较大, 可能主要是受到水动力和边界条件以及营养盐和藻类浓度的影响. 湖 心区以及西南湖区相对梅梁湾的水动力条件要更强 ${ }^{[13]}$, 因此与水动力息息相关的藻类变化过程也更加活 跃, 受到参数的影响也更强. 而梅梁湾等湖湾区由于靠近湖岸, 受到外边界输人输出河流的影响较大, 实测 值存在较大的不确定性, 模型的模拟结果对于外边界设置的精确程度较为敏感 ${ }^{[11]}$. 对于营养盐和藻类浓度 
较低的东部湖区其区间覆盖率较高 (91.67\%), 相对不确定性 (132.58\%) 也最高; 而对于较高浓度的梅梁湾 则相反, 区间覆盖率较低 (66.67\%), 相对不确定性也较小 ( 74\%) (表 3). 这说明对于营养盐和藻类浓度低的 湖区, 模拟结果的不确定性较大,需要加以关注.

模拟结果的不确定性也在随时间不断变化, 4 个湖区模拟前期的不确定性较小, 随着时间不断增加,尤 其是在 $100 \sim 150 \mathrm{~d}$ 以后, 不确定性显著增加, 根据之前的敏感性结果, 大部分敏感参数与温度和光照有关, 随着温度的升高以及光照的增强, 藻类的生长项 更加活跃,因此敏感性参数导致的结果不确定性 也随之增加.

虽然大部分实测值在不确定区间内，但有部 分实测值偏离较远. 例如, 西南湖区和梅梁湾的 部分峰值, 模拟结果低估了实际的浓度. 这可能 是河流、监测数据等存在外部不确定性因素造成 的,这些因素也是调整水质模型时不可忽视的重 要因素.

\section{3 参数取值范围}

由于本研究先验分布都是均匀分布的, 因此 可以通过参数似然值的后验分布直观地发现模 型参数的适当范围. 例如藻类最大生长速率参数 (PMc) ( 图 4), 参数取值在 2.3 2.6 的范围内, 似 然值的累计密度最大, 说明在此区间范围内模型 模拟的范围普遍较好. 而当取值大于 3.8 时, 累计 密度明显减小, 模拟结果较差, 结合其他湖区的 结果得到可能的取值范围为 $2.0 \sim 3.8$. 最终得到 7 个敏感参数的取值范围 (表 4).

表 34 个湖区不确定性指标

Tab.3 Uncertainty indicators in four subareas

\begin{tabular}{lcccc}
\hline 湖区 & $\begin{array}{c}\text { 区间 } \\
\text { 覆盖率 }\end{array}$ & $\begin{array}{c}\text { 不确定区 } \\
\text { 间宽度 }\end{array}$ & $\begin{array}{c}\text { 实测值/ } \\
\left(\mathrm{mg} / \mathrm{m}^{3}\right)\end{array}$ & $\begin{array}{c}\text { 相对不确定 } \\
\text { 区间宽度 }\end{array}$ \\
\hline 西南湖区 & $83.33 \%$ & 16.81 & 29.15 & $57.67 \%$ \\
东部湖区 & $91.67 \%$ & 16.44 & 12.40 & $132.58 \%$ \\
梅梁湾 & $66.67 \%$ & 31.96 & 43.00 & $74.33 \%$ \\
湖心区 & $75.00 \%$ & 12.34 & 23.84 & $51.76 \%$ \\
\hline
\end{tabular}

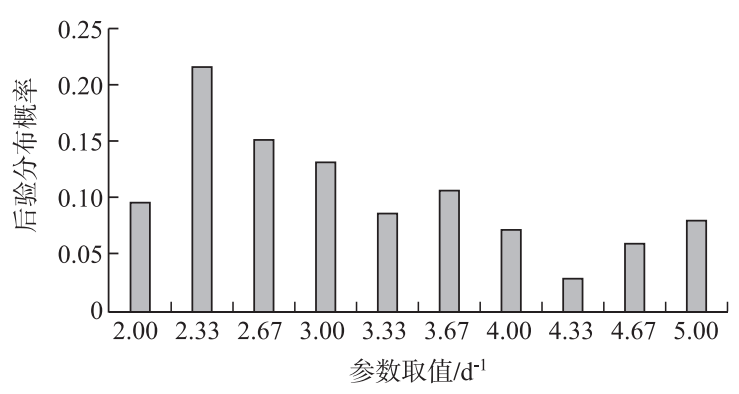

图 4 参数最大生长速率的后验分布

Fig.4 Posterior distribution of PMc

表 4 参数可能取值范围

Tab.4 Possible ranges of sensitive parameters

\begin{tabular}{ccccc}
\hline 敏感参数 & 参数意义 & 单位 & 最小值 & 最大值 \\
\hline KeChl & 叶绿素消光系数 & - & 0.04 & 0.07 \\
KTG1c & 藻类生长温度影响系数 & - & 0.006 & 0.010 \\
PRRc & 藻类牧食速率 & $\mathrm{d}^{-1}$ & 0.01 & 0.04 \\
PMc & 最大生长速率 & $\mathrm{d}^{-1}$ & 2.0 & 3.8 \\
BMRc & 藻类基础代谢速率 & $\mathrm{d}^{-1}$ & 0.02 & 0.06 \\
$\mathrm{TMc1}$ & 藻类适宜生长温度 & ${ }^{\circ} \mathrm{C}$ & 23 & 27 \\
$\mathrm{WSc}$ & 藻类沉降速率 & $\mathrm{m} / \mathrm{d}$ & 0.02 & 0.03 \\
\hline
\end{tabular}

虽然可以通过调整参数达到优化模型模拟的目的,但是由于广泛存在的其他不确定性因素,仅仅通过参 数调整无法达到最优化的目的. 并且由于参数之间存在着实际意义上的相互联系, 异参同效的现象非常普遍, 往往参数的组合比单个参数的取值更有意义,因此给出的敏感参数取值范围并不是准确的取值, 仅供参考.

\section{4 参考文献}

[ 1 ] Chen L, Yang Z, Liu H. Assessing the eutrophication risk of the Danjiangkou Reservoir based on the EFDC model. Ecological Engineering, 2016, 96: 117-127.

[ 2 ] Radwan M, Willems P, Berlamont J. Sensitivity and uncertainty analysis of river water quality modelling. Journal of Hydroinformatics, 2002, 6(2) : 83-99.

[ 3 ] Omlin M, Brun R, Reichert P. Biogeochemical model of Lake Zürich: sensitivity, identifiability and uncertainty analysis. 
Ecological Modelling, 2001, 141(1/2/3): 105-123.

[ 4 ] Arhonditsis G, Tsirtsis G, Karydis M. The effects of episodic rainfall events to the dynamics of coastal marine ecosystems: applications to a semi-enclosed gulf in the Meditteranean Sea. Journal of Marine Systems, 2002, 35(3/4) : 183-205.

[ 5 ] Li Y, Wang ZM, Zhang LJ et al. Analysis of parameter sensitivity of two-dimensional water quality model. Journal of Water Resources \& Water Engineering, 2014, 25(1): 7-12. [李燕, 王自明, 张龙江等. 二维水质模型参数的敏感性分析. 水资源与水工程学报, 2014, 25(1): 7-12.]

[ 6 ] Luo C, Li ZF, Xi Q et al. Sensitivity analysis of hydrological and water quality parameters of HSPF Model. Journal of Agro-Environment Science, 2014, 33(10): 1995-2002. [罗川, 李兆富, 席庆等. HSPF 模型水文水质参数敏感性分析. 农业环境科学学报, 2014, 33(10): 1995-2002.]

[ 7 ] Li Z, Chen Q, Xu Q. Modeling algae dynamics in Meiliang Bay of Taihu Lake and parameter sensitivity analysis. Journal of Hydro-environment Research, 2015, 9(2) : 216-225.

[ 8 ] Pastres R, Franco D, Pecenik G et al. Local sensitivity analysis of a distributed parameters water quality model. Reliability Engineering System Safety, 1997, 57(1): 21-30.

[ 9 ] Pastres R, Ciavatta S. A comparison between the uncertainties in model parameters and in forcing functions: its application to a 3D water-quality model. Environmental Modelling \& Software, 2005, 20(8) : 981-989.

[10] Yi X, Zou R, Guo H. Global sensitivity analysis of a three-dimensional nutrients-algae dynamic model for a large shallow lake. Ecological Modelling, 2016, 327: 74-84.

[11] Li YP, Qiu L, Tang CY et al. Uncertainty and sensitivity analysis of input conditions in large shallow lake hydrodynamic model. China Environmental Science, 2014, 34(2): 410-416. [李一平, 邱利, 唐春燕等. 湖泊水动力模型外部输人 条件不确定性和敏感性分析. 中国环境科学, 2014, 34(2): 410-416.]

[12] Ma RH, Kong FX, Duan HT et al. Spatio-temporal distribution of cyanobacteria blooms based on satellite imageries in Lake Taihu, China. J Lake Sci, 2008, 20(6) : 687-694. DOI:10.18307/2008.0605. [马荣华, 孔繁翔, 段洪涛等. 基 于卫星遥感的太湖蓝藻水华时空分布规律认识. 湖泊科学, 2008, 20(6) : 687-694.]

[13] Li YP, Tang CY, Yu ZB et al. Uncertainty and sensitivity analysis of large shallow lake hydrodynamic models. Advances in Water Science, 2012, 23(2): 271-277. [李一平, 唐春燕, 余钟波等. 大型浅水湖泊水动力模型不确定性和敏感性 分析. 水科学进展, $2012,23(2): 271-277$.]

[14] Li Y, Tang C, Zhu J et al. Parametric uncertainty and sensitivity analysis of hydrodynamic processes for a large shallow freshwater lake. International Association of Scientific Hydrology Bulletin, 2015: 60(6) : 1078-1095.

[15] Hornberger GM, Spear RC, Hornberger GM et al. An approach to the preliminary analysis of environmental systems. Journal of Environmental Management, 1981, 12: 1.

[16] Li Y, Wang ZM, Zhang LJ et al. Analysis of parameter sensitivity of two-dimensional water quality model. Journal of Water Resources and Water Engineering, 2014, 25(1): 7-12. [李燕, 王自明, 张龙江等. 二维水质模型参数的敏感性分析. 水资源与水工程学报. $2014,25(1): 7-12$.]

[17] Beven K, Binley A. The future of distributed models-Model calibration and uncertainty prediction. Hydrological Processes, 2010, 6(3): 279-298.

[18] Analysis EMFL. Eutrophication model for Lake Washington (USA) : Part I. Model description and sensitivity analysis. Ecological Modelling, 2005, 187(2): 140-178.

[19] Seo DI, Kim MA. Application of EFDC and WASP7 in series for water quality modeling of the Yongdam Lake, Korea. Journal of Korea Water Resources Association, 2011, 44(6) : 439-447.

[20] Wang Z, Zou R, Zhu X et al. Predicting lake water quality responses to load reduction: a three-dimensional modeling approach for total maximum daily load. International Journal of Environmental Science and Technology, 2014, 11(2): 423-436.

[21] Wang Y, Jiang Y, Liao W et al. 3-D hydro-environmental simulation of Miyun reservoir, Beijin. Journal of Hydro-environment Research, 2014, 8(4) : 383-395.

[22] Freer J, Beven K, Ambroise B. Bayesian estimation of uncertainty in runoff prediction and the value of data: An application of the GLUE approach. Water Resources Research, 1996, 32(7) : 2161-2173.

[23] Kottegoda NT, Rosso R. Statistics, probability, and reliability for civil and environmental engineers. Journal of America Chemistry Society, 1997, 10(2) : 501-503.

[24] Yi X, Zou R, Guo H. Global sensitivity analysis of a three-dimensional nutrients-algae dynamic model for a large shallow lake. Ecological Modelling, 2016, 327: 74-84.

[25] Bruce LC, Hamilton D, Imberger J et al. A numerical simulation of the role of zooplankton in C, N and P cycling in Lake Kinneret, Israel. Ecological Modelling, 2006, 193(3/4) : 412-436.

[26] Arhonditsis GB, Brett MT. Eutrophication model for Lake Washington (USA). Ecological Modelling, 2005, 187(2/3): 140-178. 